\title{
Optimal Segmentation Framework for Detection of Brain Anomalies
}

\author{
Nageswara Reddy $\mathrm{P}^{\mathrm{a},{ }^{*}}$, C.P.V.N.J.Mohan Rao ${ }^{\mathrm{b}}$, Ch.Satyanarayana ${ }^{\mathrm{c}}$ \\ ${ }^{a, *}$ Research Scholar, Jawaharlal Nehru Technological University, Kakinada, \\ ${ }^{b}$ Professor and Principal, Avanthi Institute of Engineering \& Technology \\ ${ }^{c}$ Professor, Department of computer Science and Engineering, JNTU Kakinada
}

\begin{abstract}
This work presents an enhancement in accuracy for brain disorder detection using optimal unification. The strategy for detection of segments and brain regions causing medical conditions are described. This work demonstrates the application of multilateral filter and applied watershed method with EM-GM method. The most popular existing techniques of brain tumor detection are not optimal compared to this combination of Watershed and EM-GM technique with the proposed optimal unification technique. The result is optimally unified and achieved high accuracy. The multilateral filter enhances the image edges for better segmentation using signal amplitude moderation of the pixel. In the unification process, the optimal sets of segments are divided and finest merged results are considered with the brain regions detected with anomalies. Henceforth the number of possible medical investigations will be reduced.
\end{abstract}

Index Terms: Brain MR Images, T1 Images, HMA, Watershed Method, EM-GM Method, Multilateral Filter, Optimal Unification.

(C) 2016 Published by MECS Publisher. Selection and/or peer review under responsibility of the Research Association of Modern Education and Computer Science.

\section{Introduction}

Over the last few decades, the massive growths in the brain imaging technique have explored the extended possibilities of brain anatomy analysis. In order to maximize the accuracy of detection for brain anatomy, the imaging techniques have extended the quality of the images. Thus the analysis of this complex and high quality images became the most tedious task for the technicians [1]. Moreover, due to the human intervention the investigations are bound to be erroneous. Also these manual analyses are often time-consuming and limited in finding difficulties in brain data analysis compared to the computerized methods for anomalies detection [1].

The widely used technique for analysis of medical images is segmentation based imaging in clinical analysis. For the medical purposes like analysis of brain cell distortion, regions with damaged cells, anatomical visualization and planning for brain surgery image segmentation is used. Though limitations identified from the 
study demonstrate various segmentation techniques are restricted in generating high accuracy and mostly focused in brain tumor detection. The recent researches also fail to achieve the unsurpassed accuracy [2].

Henceforth the rest of the paper is furnished with the focus to exhibit the improvement in accuracy of disorder detection for T1 type MR Images.

\section{Related Works}

The extraction of the features from the medical images makes the major outcome of the analysis. The segmentation techniques of the medical image are the most widely used for medical analysis [2]. The parallel researches have proposed multiple significant algorithms for image segmentation in 3D image visual analysis for detecting medical disorders. The researches carried out in image segmentation are classified into two major categories as supervised and unsupervised. The supervised techniques are automatic and outcomes into a high density output images and in the other hand the unsupervised techniques extracts the features and results into a feature extracted image. A wide variety of medical image segmentation techniques are been deployed to detect the brain tumors from the MR images. Some of the techniques [3] are compared here [Fig -. 1].

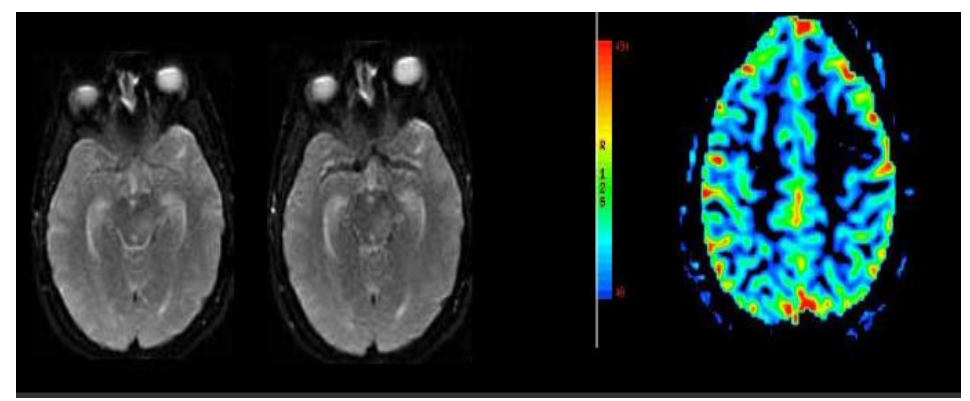

Fig.1. (a) Brain Image Analysis using Histogram
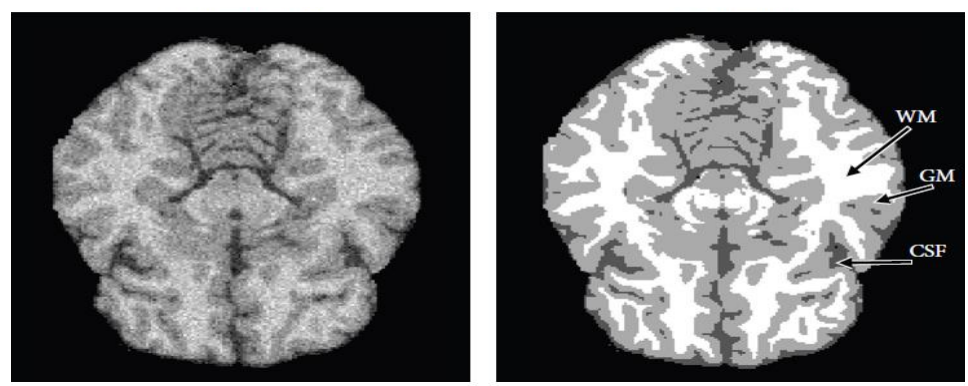

Fig.1. (b) Brain Image Analysis using Segmentation

Hence with this understanding, the next research direction is clear to have some segmentation technique to improve the possibilities of the accuracy with the scope of reduced time complexity.

Thus this work proposes an optimal unification of the results obtained from the watershed method and expectation maximization of Gaussian mixture segmentation techniques with a proposed Multilateral filtering to improve the accuracy of the brain disorder detection.

\section{Brain Anomalies Detection using the Proposed Method}

The major focus of this work is to increase the accuracy of the detection of brain anomalies for MR Images. The magnetic resonance techniques for generating the visual representation of brain images result in two 
different types of images as T1 image and T2 image. The studies demonstrate the accuracy of T1 images is higher for detecting the anomalies. Hence in this work we focus on T1 images to carry out the proposed method. The core framework is been demonstrated here [Fig. 2].

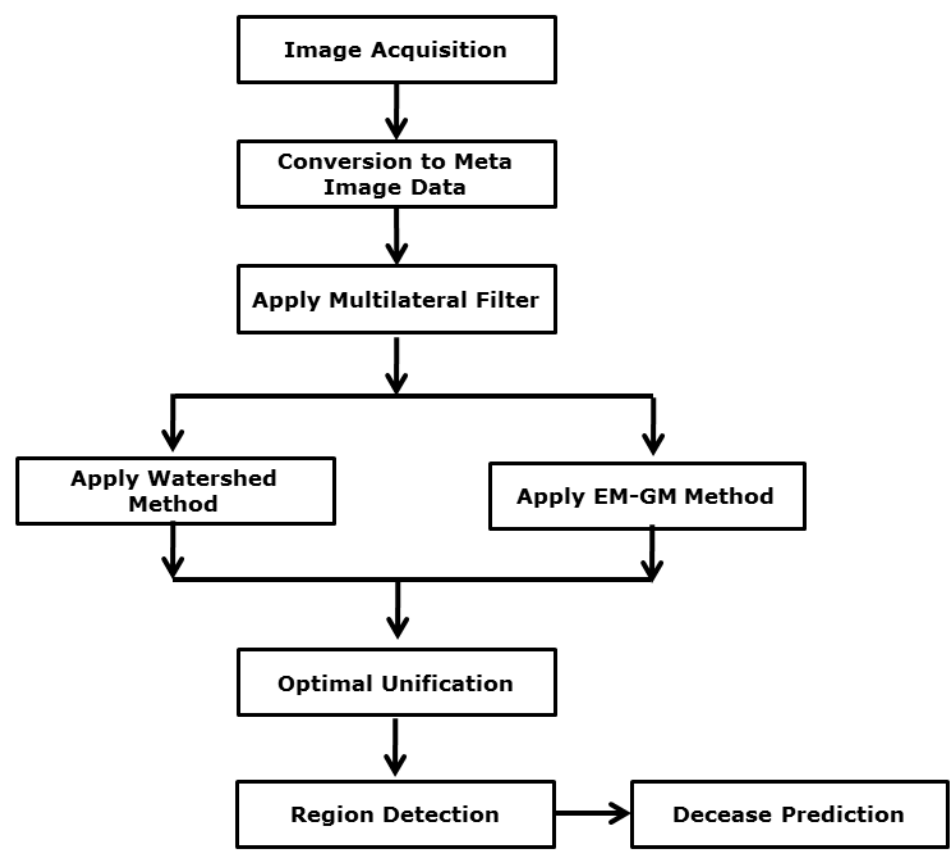

Fig.2. Proposed framework for Brain Anomaly Detection

\subsection{Proposed Multilateral Filter}

The Proposed multilateral filter is based on the existing bilateral filter for improving the input image variance and standard deviation [4] [11].

The bilateral filter explained as

$$
\overline{I M G}\left(C o_{1}\right)=\frac{1}{N\left(C o_{1}\right)} \sum_{C o_{1} \in P x} I M G\left(C o_{2}\right) \cdot g\left(C o_{1}, C o_{2}\right) \cdot P\left(I M G\left(C o_{2}\right), I M G\left(C o_{1}\right)\right)
$$

Where,

$I M G_{\text {denotes the original image, }} \overline{I M G}$, denotes the filtered and noise removed image, ${ }^{C o_{1}}$ and $C o_{2}$, denotes the spatial coordinates of the image, Px, denotes the collection of pixels around the noise, $N\left(\mathrm{Co}_{1}\right)$, denotes the normalization constant for each pixel to restrict the value after normalization within geometric and photonic range denoted by Px and $g$ and $p$, denotes the geometric and photometric similarities of the image.

Hence the enhancement of the image is proposed to regularize the local signal amplitude of every pixel value:

$$
\overline{I M G}\left(C o_{1}\right)=\frac{1}{N\left(C o_{1}\right)} \sum_{C o_{1} \in P x} I M G\left(C o_{2}\right) \cdot \theta\left(C o_{1}, C o_{2}, t\right)
$$


As,

$$
\begin{aligned}
& \theta\left(\mathrm{Co}_{1}, \mathrm{Co}_{2}, t\right)= \\
& \left(1-a\left(C o_{1}\right)\right) \cdot g\left(\mathrm{Co}_{1}, C o_{2}\right) \\
& +a\left(C o_{1}\right) \cdot g\left(C o_{1}, C o_{2}\right) \cdot P\left(I M G\left(C o_{1}\right), I M G\left(C o_{2}\right)\right) \cdot \sum_{i=1}^{D-1} d_{i}\left(C o_{1}, C o_{2}\right)
\end{aligned}
$$

Where,

$a\left(C o_{1}\right)$, denotes the regularized local signal amplitude of the pixel, $\mathrm{d}_{\mathrm{i}}$, denotes the image dimensions for during noise removal.

The improvement in the input images are been recorded [Table -1] and the improvement in variance and standard deviation is been observed.

Table 1. Improvement in the Input Data by Multilateral Filter

\begin{tabular}{ccccccc}
\hline $\begin{array}{c}\text { Image } \\
\text { Dataset } \\
\text { In MHA }\end{array}$ & $\begin{array}{c}\text { Actual Image } \\
\text { Variance }\end{array}$ & $\begin{array}{c}\text { Filtered Image } \\
\text { Variance }\end{array}$ & Improvement & $\begin{array}{c}\text { Actual Image } \\
\text { Std. Deviation }\end{array}$ & $\begin{array}{c}\text { Filtered Image } \\
\text { Std. Deviation }\end{array}$ & Improvement \\
\hline Dataset 1 & 4426745 & 19162216 & 3.3 & 72 & 104 & 0.44 \\
Dataset 2 & 8406091 & 26021378 & 2 & 74 & 99 & 0.33 \\
Dataset 3 & 16958827 & 19501273 & 0.14 & 100 & 103 & 0.03 \\
Dataset 4 & 17772628 & 19802241 & 0.11 & 99 & 102 & 0.03 \\
Dataset 5 & 13768559 & 14906214 & 0.08 & 93 & 95 & 0.02 \\
\hline
\end{tabular}

\section{Applied Watershed Method}

The applied watershed [3] [12] [13] method is described as morphological gradient-based segmentation for this work [Fig.3]. The minimal watershed method is:

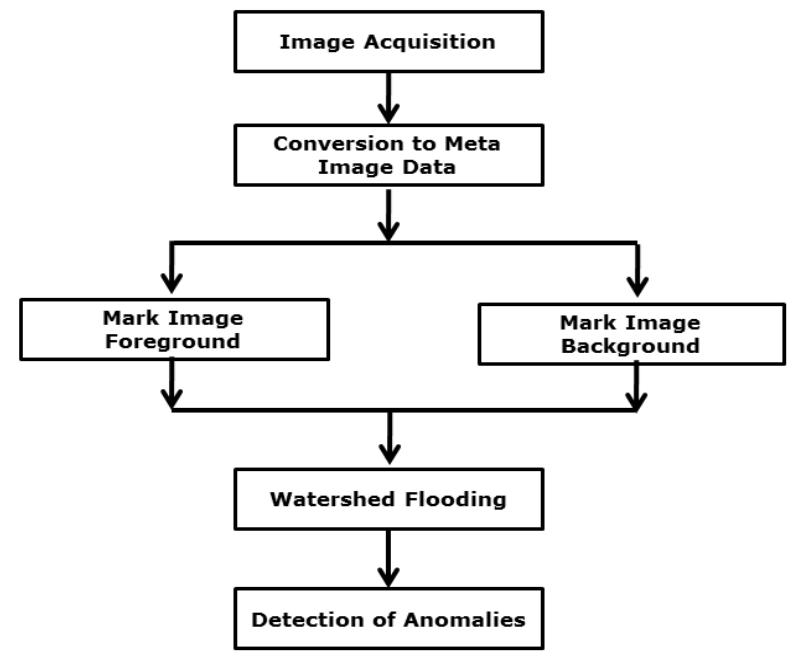

Fig.3. Applied Watershed Method Framework 
Considering the $\beta(a, b)$ with $(a, b) \in I^{2}$ as a scalar representation of the image IMG, hence the morphological gradient is

$$
\partial_{M} \beta=(\beta \oplus M)-(\beta \varnothing M)
$$

Here,

$(\beta \oplus M)$ denotes the dilation, $(\beta \varnothing M)$ denotes the erosion and $\mathrm{M}$ denotes the structuring element.

Hence the Morphological Laplacian is

$$
\Delta_{M} \beta=(\beta \oplus M)-(\beta \varnothing M)
$$

Hence, the segments to be classified as maxima can be identified as

$$
\Delta_{M} \beta<0
$$

And the segments to be classified as minima can be identified as

$$
\Delta_{M} \beta>0
$$

Thus the edge of the segments can be identified as

$$
\Delta_{M} \beta=0
$$

Henceforward with the maxima, minima and edges now the watershed method can recursively identify the segments in the image.

The Applied Watershed Method detects the brain anomalies [Fig.4].

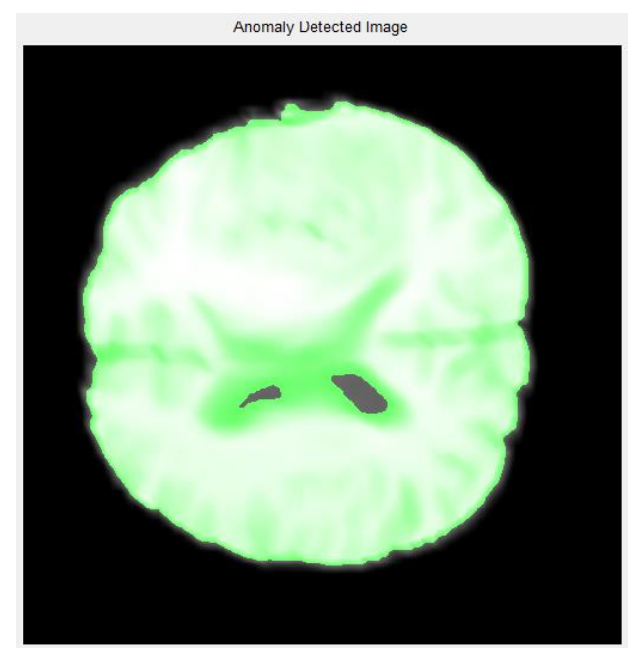

Fig.4. Applied Watershed Method Results 
Applied Expectation Maximization and Gaussian Mixture Method

The Gaussian mixture method [Fig.5] [5] [6] [10] for each pixel value for input image is described as:

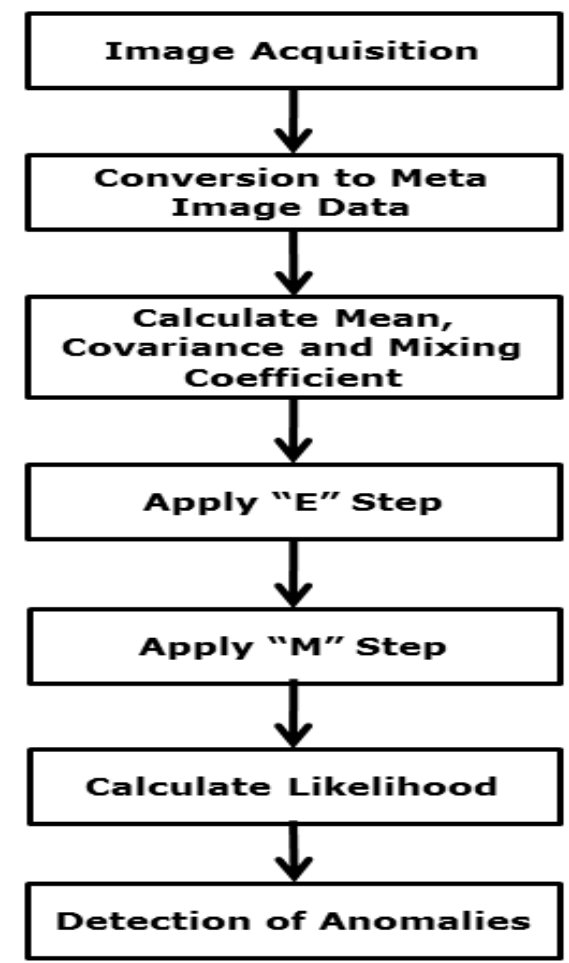

Fig.5. Applied Gaussian Mixture Method Framework

$$
G(i)=N(i \mid \mu, \Sigma)
$$

Where

$\mathrm{N}$ denotes the Gaussian normal distribution, $\mu$ denotes the mean and $\Sigma$ denotes the variance

The inclusion of the multiple neighboring pixel will result in

$$
G(i)=N\left(i \mid \mu_{1}, \Sigma_{1}\right)+N\left(i \mid \mu_{2}, \Sigma_{2}\right)
$$

Henceforth, applicability of mixing coefficient will result in

$$
G(i)=\pi_{1} N\left(i \mid \mu_{1}, \Sigma_{1}\right)+\pi_{2} N\left(i \mid \mu_{2}, \Sigma_{2}\right)
$$

Thus the Gaussian mixture for the complete image will result in 
$G(i)=\sum_{z=1}^{K} \pi_{z} N\left(i \mid \mu_{z}, \Sigma_{z}\right)$

Where $\mathrm{K}$ denotes total number of pixels in the input image

After application of Gaussian mixture method, the expectation maximization needs to be applied Expectation step as score for each pixel:

$$
i: \gamma_{z}
$$

Then, the Gaussian parameters to be mapped into the score point:

$$
\pi_{z}, \mu_{z}, \Sigma_{z} \rightarrow \gamma_{z}
$$

And Finally, the likelihood to be calculated to converge.

The Applied EM-GM Method detects the brain anomalies [Fig.6].

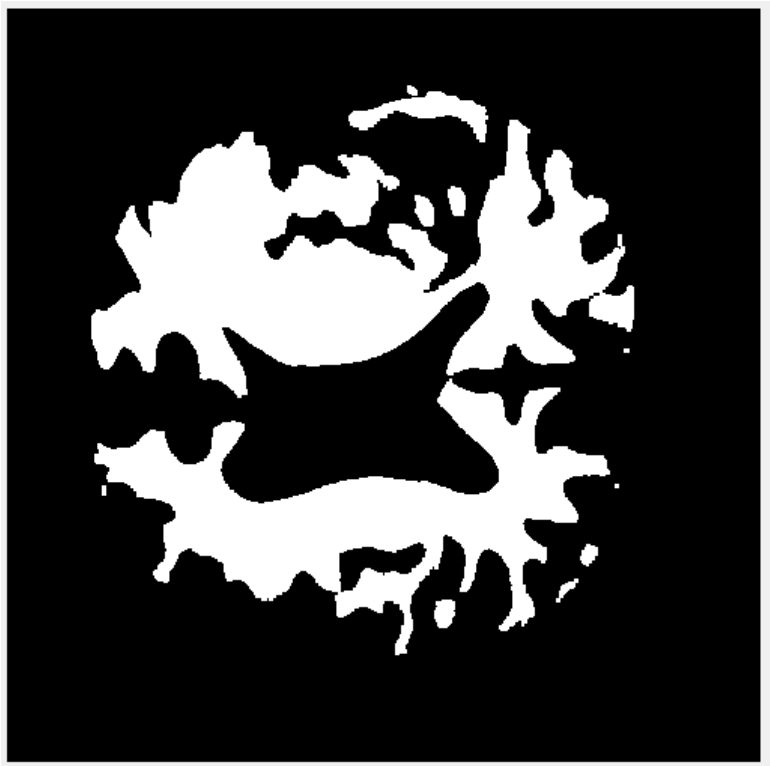

Fig.6. Applied EM-GM Method Results

\section{Proposed Optimal Unification}

The optimal unification of the result images are carried out in two phases. In the first phase, the images are been divided into segments and in the next phase from the input images the segments are been merged. The approach is demonstrated here:

Optimal Segment Dividing Algorithm: Image1 and Image2 are the results from Watershed and EM-GM method respectively 
Step-1.1. Calculate the number of regions for Image1 and Image 2 and store in $\mathrm{n}$ and $\mathrm{m}$ respectively

$\Psi\left[I M G_{1}\right] \rightarrow n, \Psi\left[I M G_{2}\right] \rightarrow m$

Step-1.2. Repeat Till $n+m$, where $m=n$ denotes the size of the SegmentList arrays

$\forall\left\{\Psi\left[I M G_{1}\right], \Psi\left[I M G_{2}\right]\right\}$

Step-1.3. Compare All SegmentList1[n] and SegmentList2[m] to find the unique region

$\exists\left[\forall\left\{\Psi\left[I M G_{1}\right]\right\}\right]_{\forall ! n_{i} \neq n_{j}}, \exists\left[\forall\left\{\Psi\left[I M G_{2}\right]\right\}\right]_{\forall ! m_{i} \neq m_{j}}$

Step-1.4. If SegmentList1[n] and SegmentList2[m] are unique, then Store the segmented regions into the SegmentList1, SegmentList2 from Image1 and Image2 respectively

$\exists\left[\forall\left\{\Psi\left[I M G_{1}\right]\right\}\right]_{\forall ! n_{i} \neq n_{j}} \rightarrow$ SegmentList

$\exists\left[\forall\left\{\Psi\left[I M G_{2}\right]\right\}\right]_{\forall ! m_{i} \neq m_{j}} \rightarrow$ Segment List $_{2}$

Optimal Segment Combining Algorithm: SegmentList1, SegmentList2 are obtained from the Eq. 13

Step-2.1. Calculate the number of regions for SegmentList1, SegmentList2 and store in $\mathrm{n}$ and $\mathrm{m}$ respectively

Step-2.2. Repeat Till $n+m$, where $m=n$ denotes the size of the SegmentList arrays

Step-2.3. Compare each Segment from SegmentList1 and SegmentList2 to find the similar regions

Step-2.4. If the regions are nearing neighbors, then combine the regions

Step-2.5. Mark the regions with anomalies

The proposed method identifies the region for disease prediction [Fig.7].

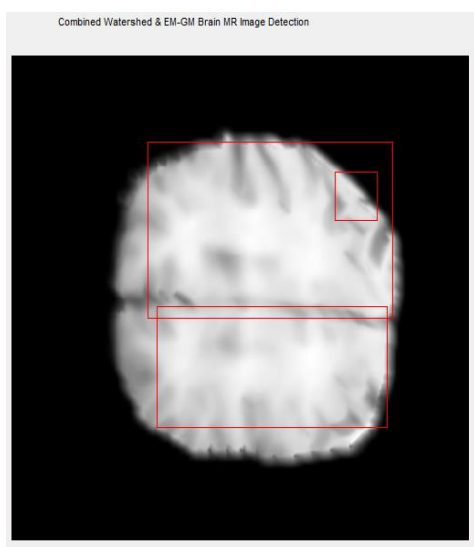

Fig.7. Optimal Unification Method Results 
Based on the region marks, this technique will predict the possible diseases [Table - 2] [9] [7] [8].

Table 2. Brain Anomalous Area and Prediction of Diseases

\begin{tabular}{cc}
\hline Brain Region & Predictable Diseases \\
\hline Amygdala & Memory Loss, Anxiety, Phobia, Post - Traumatic Disorder \\
Prefrontal Cortex & Stress \\
Anterior Cingulate Cortex & ADHD, Schizophrenia, Depression \\
Hippocampus & Mood Disorder \\
\hline
\end{tabular}

\section{Results and Discussion}

The applied Watershed [Table - 3] and EM-GM [Table - 4] method is been applied on the dataset to compare the achieved results of the Optimal Segmentation Technique [Table - 5] and achieved a comparative result improvement.

Table 3. Accuracy Analysis of Watershed Method

\begin{tabular}{cccc}
\hline $\begin{array}{c}\text { Image Dataset } \\
\text { In MHA format }\end{array}$ & $\begin{array}{c}\text { Number of Images in the } \\
\text { Dataset }\end{array}$ & $\begin{array}{c}\text { Number of Correctly } \\
\text { Identified Images }\end{array}$ & $\begin{array}{c}\text { Accuracy (\%) for } \\
\text { Identified Images }\end{array}$ \\
\hline Dataset 1 & 10 & 6 & 98.13 \\
Dataset 2 & 10 & 8 & 98.12 \\
Dataset 3 & 10 & 6 & 97.99 \\
Dataset 4 & 10 & 8 & 98 \\
Dataset 5 & 10 & 6 & 95.36 \\
Dataset 6 & 10 & 7 & 92.69 \\
Dataset 7 & 10 & 6 & 97.98 \\
Dataset 8 & 10 & 6 & 97.98 \\
Dataset 9 & 10 & 8 & 85.43 \\
Dataset 10 & 10 & 6 & 97 \\
\hline
\end{tabular}

The obtained average accuracy is $95.868 \%$, the mean accuracy is $95.868 \%$ and the median accuracy is 97.98\% for Watershed Method.

Table 4. Accuracy Analysis of Expectation Maximization - Gaussian Mixture Method

\begin{tabular}{cccc}
\hline $\begin{array}{c}\text { Image Dataset } \\
\text { In MHA format }\end{array}$ & $\begin{array}{c}\text { Number of Images in the } \\
\text { Dataset }\end{array}$ & $\begin{array}{c}\text { Number of Correctly } \\
\text { Identified Images }\end{array}$ & $\begin{array}{c}\text { Accuracy (\%) for } \\
\text { Identified Images }\end{array}$ \\
\hline Dataset 1 & 10 & 7 & 96.97 \\
Dataset 2 & 10 & 8 & 98.12 \\
Dataset 3 & 10 & 8 & 91.53 \\
Dataset 4 & 10 & 7 & 91.67 \\
Dataset 5 & 10 & 6 & 95.63 \\
Dataset 6 & 10 & 8 & 93.04 \\
Dataset 7 & 10 & 7 & 94.25 \\
Dataset 8 & 10 & 7 & 92.03 \\
Dataset 9 & 10 & 7 & 85.43 \\
Dataset 10 & 10 & 7 & 97.54 \\
\hline
\end{tabular}


The obtained average accuracy is $93.621 \%$, the mean accuracy is $93.621 \%$ and the median accuracy is 93.645\% for EM-GM Method.

Table 5. Accuracy Analysis of Proposed Optimal Unification Method

\begin{tabular}{cccc}
\hline $\begin{array}{c}\text { Image Dataset } \\
\text { In MHA format }\end{array}$ & $\begin{array}{c}\text { Number of Images in the } \\
\text { Dataset }\end{array}$ & $\begin{array}{c}\text { Number of Correctly } \\
\text { Identified Images }\end{array}$ & Accuracy (\%) \\
\hline Dataset 1 & 10 & 8 & 99.13 \\
Dataset 2 & 10 & 9 & 99.12 \\
Dataset 3 & 10 & 10 & 98.99 \\
Dataset 4 & 10 & 8 & 99 \\
Dataset 5 & 10 & 9 & 96.65 \\
Dataset 6 & 10 & 9 & 98.04 \\
Dataset 7 & 10 & 8 & 98.98 \\
Dataset 8 & 10 & 9 & 86.43 \\
Dataset 9 & 10 & 8 & 98.54 \\
Dataset 10 & 10 & 10 & \\
\hline
\end{tabular}

The obtained average accuracy is $96.986 \%$, the mean accuracy is $96.986 \%$ and the median accuracy is $99 \%$ in the proposed method.

Hence this work shows significant improvement of accuracy for all the tested datasets for 300 patients [Fig.8].

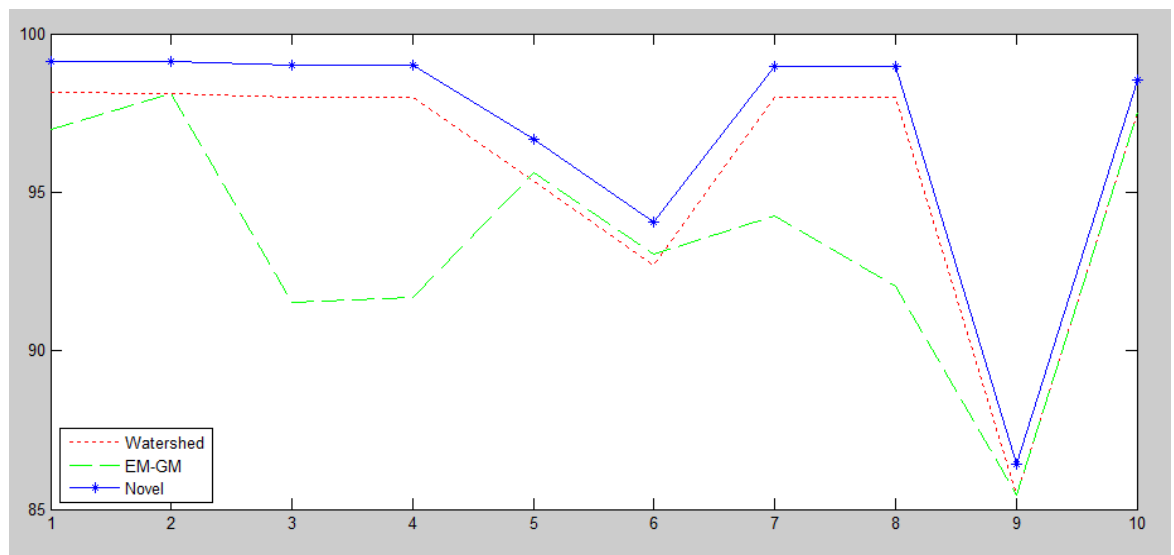

Fig.8. Accuracy Comparison of Applied Watershed Method, Applied EM-GM Method and Optimal Unification Method

\section{Conclusion}

Quantitative analysis of brain MR images allows a greater understanding of the nature of the diseases. The proposed algorithm in this work has been tested on BRATS 2012 (Nice), BRATS 2013 (Nagoya) and BRATS 2014 (Boston) challenge datasets and demonstrates higher accuracy. The work also concludes the optimal technique for medical image segmentation and detection of brain anomalies. Compared to the existing research outcomes, this work proposes the mapping of possible disease with the brain anomalous regions. With the final outcome of accuracy improvement and disease prediction, the work certainly and satisfyingly extends the possibilities of better medical image processing. 


\section{References}

[1] N. Porz, "Multi-modalodal glioblastoma segmentation: Man versus machine", PLOS ONE, vol. 9, pp. e96873, 2014.

[2] S. Bauer, R. Wiest, L.-P. Nolte and M. Reyes, "A survey of MRI-based medical image analysis for brain tumor studies", Phys. Med. Biol., vol. 58, no. 13, pp. R97-R129, 2013.

[3] D. W. Shattuck, G. Prasad, M. Mirza, K. L. Narr and A. W. Toga, "Online resource for validation of brain segmentation methods", Neuroimage, vol. 45, no. 2, pp. 431-439, 2009.

[4] M. Stille, M. Kleine ; J. Hagele ; J. Barkhausen ; T. M. Buzug, "Augmented Likelihood Image Reconstruction", IEEE Transactions on Medical Imaging, Volume:35, Issue:1.

[5] A. Gooya, G. Biros and C. Davatzikos, "Deformable registration of glioma images using EM algorithm and diffusion reaction modeling", IEEE Trans. Med. Imag., vol. 30, no. 2, pp. 375-390, 2011.

[6] L. Weizman, "Automatic segmentation, internal classification, and follow-up of optic pathway gliomas in MRI", Med. Image Anal., vol. 16, no. 1, pp. 177-188, 2012.

[7] K. Hameeteman, "Evaluation framework for carotid bifurcation lumen segmentation and stenosis grading", Med. Image Anal., vol. 15, no. 4, pp. 477-488, 2011.

[8] S. Ahmed, K. M. Iftekharuddin and A. Vossough, "Efficacy of texture, shape, and intensity feature fusion for posterior-fossa tumor segmentation in MRI", IEEE Trans. Inf. Technol. Biomed., vol. 15, no. 2, pp. 206-213, 2015.

[9] R. Achanta et al., "SLIC superpixels compared to state-of-the-art su- perpixel methods," IEEE Trans. Pattern Anal. Mach. Intell., vol. 34, no. 11, pp. 2274-2282, Nov. 2012.

[10] B. B. Avants et al., "A reproducible evaluation of ANTs similarity metric performance in brain image registration," Neuroimage, vol. 54, no. 3, pp. 2033-44, Feb. 2011.

[11] N. Subbanna, D. Precup, L. Collins, and T. Arbel, "Hierarchical prob- abilistic Gabor and MRF segmentation of brain tumours in MRI vol- umes," Proc. MICCAI, vol. 8149, pp. 751-758, 2013.

[12] H. C. Shin, M. R. Orton, D. J. Collins, S. J. Doran, and M. O. Leach, "Stacked autoencoders for unsupervised feature learning and multiple organ detection in a pilot study using 4D patient data," IEEE Trans. Pattern Anal. Mach. Intell., vol. 35, no. 8, pp. 1930-1943, Aug. 2013.

[13] A. Islam, S. M. S. Reza, and K. M. Iftekharuddin, "Multi-fractal texture estimation for detection and segmentation of brain tumors," IEEE Trans. Biomed. Eng., vol. 60, no. 11, pp. 3204-3215, Nov. 2013.

\section{Authors' Profiles}

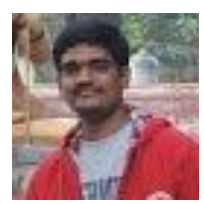

P. Nageswara Reddy is a Research Scholar of Department of Computer Science and Engineering, Jawaharlal Nehru Technological University, Kakinada. He has 9 years of Software Industry and Teaching experiences for Graduate and Post Graduate engineering courses. His current research interests are Data Warehousing, Image Processing and Cloud Computing.

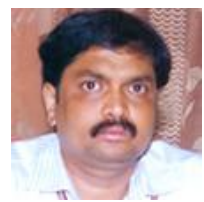

Dr. C. P. V. N. J Mohan Rao is Professor in the Department of Computer Science and Engineering, Avanthi Institute of Engineering \& Technology - Narsipatnam. He did his PhD from Andhra University and his research interests include Image Processing, Wireless Networks and Information Security. He has guided more than 50 M.Tech Projects and currently guiding four research scholars for Ph.D. He received many honours and he has been the member for many expert committees, member of many professional bodies and Resource person for various 
organizations.

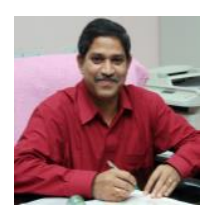

Dr. Ch. Satyanarayana is a Professor in Department of Computer Science and Engineering at Jawaharlal Nehru Technological University Kakinada. He completed B. Tech and M.Tech in Computer Science and Engineering from Andhra University, Visakha Patnam, Andhra Pradesh. He was awarded his Doctoral degree in 2008 from J.N.T. University, Hyderabad. He has 15 years of experience. His areas of interest are Image Processing, Databases, Pattern Recognition and Network Security. He published more than 21 research papers in International Journals and more than 100 research papers in International Conferences. He has guided 15 Research Scholars are working on different areas like Image Processing, Speech Recognition, and Pattern Recognition. He has guided more than 78 M.Tech Projects, 56 MCA Projects, and 36 B.Tech Projects.

How to cite this paper: Nageswara Reddy P, C.P.V.N.J.Mohan Rao, Ch.Satyanarayana,"Optimal Segmentation Framework for Detection of Brain Anomalies", International Journal of Engineering and Manufacturing(IJEM), Vol.6, No.6, pp.26-37, 2016.DOI: 10.5815/ijem.2016.06.03 